\title{
La discriminación de género en la relación laboral: igualdad formal, igualdad material y acción positiva*
}

\author{
Elisa Sierra Hernáiz**
}

\section{RESUMEN}

Uno de los mayores retos que se plantea en el actual marco de relaciones laborales es la aplicación de las medidas de acción positiva en cuanto tratamientos preferenciales a favor de las trabajadoras para superar los efectos de la discriminación social. La principal dificultad radica en la delimitación que de las mismas hace el ordenamiento jurídico comunitario europeo, como una excepción al principio de igualdad de trato y de igualdad de oportunidades formal, lo que ha introducido elementos ajenos a su naturaleza, siguiendo la doctrina del Tribunal de Justicia de la Unión Europea, con todo lo que ello supone de pérdida en la lucha para lograr la igualdad de oportunidades real entre los sexos en el ámbito laboral.

PALABRAS ClAVE: discriminación laboral sexos; acción positiva; tratamientos preferenciales; igualdad real; interpretación jurisprudencial.

\section{ABSTRACT}

One of biggest problems of female workers is to get real equality in their working conditions. Affirmative actions or preferential treatment can be a solution to overcome the effects of social discrimination at work. Nowdays the main obstacle to set up is not the legal delimitation in the European Law but the interpretation of cases law that limit its application in employment contracts. This situation represents a loss in the struggle for equal opportunity and rights for female works.

KEYWORDS: gender discrimination at work; affirmative action; preferential treatment; real equality; cases law.

* $\quad$ Este artículo ha sido desarrollado dentro del marco del Proyecto I+D DER 2013-42759R "Derecho del trabajo y crisis económica (II): las respuestas a la negociación colectiva", financiado por el Ministerio de Economía y Competitividad de España.

** Titular de Derecho del Trabajo y de la Seguridad Social en la Universidad Pública de Navarra. 


\section{INTRODUCCIÓN}

$\mathrm{U}$ na de las cuestiones más controvertidas a las que se ha tenido que enfrentar el derecho del trabajo en los últimos años es admitir o no la legitimidad de las medidas de acción positiva a favor de las trabajadoras para garantizar su igualdad de oportunidades en el mercado de trabajo y superar, de esta manera, las situaciones de discriminación y segregación profesional que les impiden disfrutar de unas verdaderas condiciones de igualdad en el mercado laboral. ${ }^{1}$ En la actualidad, el derecho social comunitario europeo y, por ende, el derecho español, sí que las aceptan desde un punto de vista legal aunque en la realidad práctica son de muy difícil implantación por los requisitos exigidos por la doctrina del Tribunal de Justicia de la Unión Europea (TJUE). ${ }^{2}$ La explicación última de esta situación puede tener su origen en las suspicacias que levantan su implantación por cuestionar el derecho fundamental a la igualdad y la prohibición de discriminación por razón de sexo en su afección más tradicional. Así, la finalidad de las medidas de acción positiva es dar un puesto de trabajo a una trabajadora en vez de a un trabajador sobre la base de un factor de diferenciación prohibido -el sexo- como forma de garantizar la plena igualdad de oportunidades en el mercado laboral de las mujeres, cumpliendo con los mandatos del principio de igualdad material, siempre y cuando respeten el juicio de igualdad desde los principios de razonabilidad, proporcionalidad y objetividad. Teniendo en cuenta, pues, la importancia de los bienes jurídicos protegidos se hace necesario un estudio de las mismas para delimitar claramente a qué se hace referencia cuando se habla de acción positiva y de las implicaciones que dichas medidas conllevan para garantizar que su correcta implantación.

1. En general, sobre el papel de las medidas de acción positiva en el ámbito laboral, véase Natividad Mendoza Navas, "Acciones positivas: instrumento para eliminar las diferencias entre hombres y mujeres en el ámbito de las relaciones laborales", en Teresa Martín López, dir., La igualdad de género desde la perspectiva social, jurídica y económica (Madrid: Civitas, 2014), 180 y s. En cuanto a la aplicación del principio de igualdad y no discriminación en el ámbito laboral véase José Fernando Lousada Arochena, $E l$ derecho fundamental a la igualdad efectiva de mujeres y hombres: fundamentos del derecho a la igualdad de género y, en especial, su aplicación en el derecho del trabajo y de la seguridad social (Valencia: Tirant lo Blanch, 2014).

2. Sobre la evolución de la doctrina del TJUE, véase Rosa María Giles Carnero, "La jurisprudencia del Tribunal de Justicia en materia de igualdad entre hombres y mujeres. Especial referencia al concepto de acción positiva", en M. Mora-Ruiz, dir., Formación y objeto del derecho antidiscriminatorio de género: perspectiva sistemática de la igualdad desde el Derecho público (Barcelona: Atelier, 2010), 55 y s. 


\section{DELIMITACIÓN CONCEPTUAL DE LOS CONCEPTOS DE IGUALDAD Y NO DISCRIMINACIÓN}

\section{A) El PRINCIPIO DE IGUALdAd Y SUS MANIFESTACIONES}

El principio de igualdad está integrado por dos vertientes: la igualdad formal o igualdad de trabajo y la igualdad sustancial o igualdad real o material. Respecto a la primera de ellas, en el ordenamiento jurídico español el artículo 14 de la Constitución Española (CE) señala: "Los españoles son iguales antes la Ley, sin que pueda prevalecer discriminación alguna por razón de nacimiento, raza, sexo, religión, opinión o cualquier otra condición o circunstancia personal o social".

Así delimitado, este artículo consagra el derecho que todo ciudadano tiene a obtener un trato igual -lo que es conocido como principio de igualdad formal de tratopor parte de los poderes públicos sin que puedan establecerse diferencias arbitrarias entre las personas fundadas en estos criterios. Además, es un derecho de titularidad individual, lo que significa que a nadie le podrá ser negado por la mera pertenencia a un colectivo o por una opción personal vinculada a la dignidad humana. Ello supone, como regla general de aplicación, la prohibición de los tratamientos discriminatorios, entendiendo por tales las conductas peyorativas basadas precisamente en condiciones u opciones personales sobre las que una persona no tiene facultad de elección por ser innata a ella o bien por tratarse del ejercicio de un derecho personal, asociado en la mayoría de las ocasiones al ejercicio de la libertad como derecho fundamental. ${ }^{3}$

Pero también forma parte del principio de igualdad su vertiente sustancial, real o material que, en el ordenamiento jurídico español, queda recogido en el artículo 9.2 CE: "Corresponde a los poderes públicos promover las condiciones para que la libertad y la igualdad del individuo y de los grupos en que se integra sean reales y efectivas; remover los obstáculos que impidan o dificulten su plenitud y facilitar la participación de todos los ciudadanos en la vida política, económica, cultural y social". En dicho precepto se establece una obligación-mandato a los poderes públicos de promover las condiciones para que la igualdad del individuo y de los grupos que integran la sociedad sean reales y efectivas, y remover los obstáculos que impidan o dificulten su plenitud. Su finalidad es acabar con las situaciones históricas de discriminación de determinados colectivos, corrigendo las desigualdades sociales, lo que implica una actividad promocional por parte del Estado, tanto positiva -promoción

3. Por todos, véase Miguel Rodríguez-Piñero y María Fernanda Fernández López, Igualdad y discriminación (Madrid: Tecnos, 1986). 
de condiciones para que la igualdad sea real y efectiva- como negativa-remoción de obstáculos que impidan el logro de esa igualdad-. ${ }^{4}$

La importancia de este artículo fue consagrada en la Sentencia del Tribunal Constitucional Español (TC) 128/1987, de 16 de julio, ${ }^{5}$ que defiende una tutela antidiscriminatoria unilateral con un sentido emancipador y corrector para acabar con situaciones de discriminación social de grupos preteridos, lo que legitima un derecho desigual igualatorio cuyo objetivo sea restablecer la igualdad socialmente ignorada:

pero ello no debe hacer olvidar que, por un lado, y como reiteradamente ha indicado este Tribunal, no toda desigualdad de trato resulta contraria al principio de igualdad, sino aquella que se funda en una diferencia de supuestos de hecho injustificados de acuerdo con criterios o juicios de valor generalmente aceptados, y por otro, que, como este mismo Tribunal ha sostenido, el tratamiento diverso de situaciones distintas "puede incluso venir exigido, en un Estado social y democrático de Derecho, para la efectividad de los valores que la Constitución consagra con el carácter de superiores del ordenamiento, como son la justicia y la igualdad (art. 1), a cuyo efecto atribuye además a los poderes públicos el que promuevan las condiciones para que la igualdad sea real y efectiva". La actuación de los poderes públicos para remediar, así, la situación de determinados grupos sociales definidos, entre otras características, por el sexo (y, cabe afirmar, en la inmensa mayoría de las veces, por la condición femenina) y colocados en posiciones de innegable desventaja en el ámbito laboral, por razones que resultan de tradiciones y hábitos profundamente arraigados en la sociedad y difícilmente eliminables, no puede considerarse vulneradora del principio de igualdad, aun cuando establezca para ellas un trato más favorable, pues se trata de dar tratamiento distinto a situaciones efectivamente distintas.

Desde este perspectiva, se impone que, a la hora de valorar la racionalidad y proporcionalidad de ciertas medidas y disposiciones -valoración que es necesaria desde el punto de vista del juicio de igualdad-como puedan ser las medidas de acción positiva, la existencia de colectivos sometidos a condiciones especialmente desventajosas y sí la medida analizada pretende poner fin a esta situación. ${ }^{6}$

4. Elisa Sierra Hernáiz, Acción positiva y empleo de la mujer (Madrid: CES, 1999), 9 y s.

5. Sobre la evolución de la doctrina del Tribunal Constitucional Español en materia de igualdad y no discriminación en el ámbito de las relacionales, véase Magdalena Nogueira Guastivino, "El principio de igualdad y no discriminación en las relaciones laborales: perspectiva constitucional reciente", Lan Harremanak: Revista de Relaciones Laborales, No. 25 (2011): 23 y s.

6. Véase Gemma Fabregat Monfort, Las medidas de acción positiva: la posibilidad de una nueva tutela antidiscriminatoria (Valencia: Tirant lo Blanch, 2009). 


\section{B) LA PROHIBICIÓN DE DISCRIMINACIÓN Y SUS MANIFESTACIONES}

Al igual que el principio de igualdad, la prohibición de discriminación posee dos manifestaciones dependiendo de a qué concepto de igualdad hagamos referencia. Así, al principio de igualdad formal o de trato consagrado en el artículo 14 de la CE van unidos los conceptos de discriminación directa e indirecta. A diferencia de lo que sucede con el principio de igualdad, el legislador sí que define ambos conceptos, siendo la referencia principal el derecho comunitario europeo, en concreto la Directiva $54 / 2006$, de 5 de julio, relativa a la aplicación del principio de igualdad de oportunidades e igualdad de trato entre hombres y mujeres en asuntos de empleo y ocupación que en su artículo 4.1 establece que: ${ }^{7}$

1. A efectos de la presente Directiva se entenderá por:

a) "discriminación directa": la situación en que una persona sea, haya sido o pudiera ser tratada por razón de sexo de manera menos favorable que otra en situación comparable;

b) "discriminación indirecta": la situación en que una disposición, criterio o práctica aparentemente neutros sitúan a personas de un sexo determinado en desventaja particular con respecto a personas del otro sexo, salvo que dicha disposición, criterio o práctica pueda justificarse objetivamente con una finalidad legítima y que los medios para alcanzar dicha finalidad sean adecuados y necesarios.

En el ordenamiento jurídico español el artículo 6 de la Ley Orgánica 3/2007, de 22 de marzo, para la igualdad efectiva de mujeres y hombres (LOI), delimita ambos conceptos de una manera idéntica a la Directiva Comunitaria, siendo importante destacar el papel que la jurisprudencia comunitaria ha tenido en esta materia ya que fue, por un lado, la encargada de delimitar su contenido hasta aprobación de la Directiva y, por otro, por su influencia directa en los diversos ordenamientos nacionales comunitarios. ${ }^{8}$

7. María Josefina Mateu Carruana, "Igualdad de trato y no discriminación entre mujeres y hombres en el empleo: significado y alcance de la Directiva 2006/54/CE", en A. Sempere Navarro y M. Areta Martínez, Cuestiones actuales sobre derecho social comunitario (Murcia: Laborum, 2009), 253 y s.

8. En España es de destacar la Sentencia del TC 145/1991, de 1 de julio, ya que fue la primera vez que se delimitaron los conceptos de discriminación directa e indirecta. En concreto merece destacar el fundamento jurídico 2o. que afirma: "La prohibición constitucional de discriminación por características personales y en particular por el sexo, como signo de pertenencia de la mujer a un grupo social determinado objeto históricamente de infravaloración social, económica y jurídica, se conecta también con la noción sustancial de igualdad. Ello permite ampliar y enriquecer la propia noción de discriminación, para incluir no solo la noción de discriminación directa, o sea, un tratamiento diferenciado perjudicial en razón del sexo donde el sexo sea objeto de consideración directa, sino también la noción de discriminación indirecta, que incluye los tratamientos formalmente no discriminatorios de los que derivan, por las diferencias fácticas que tienen lugar entre trabajadores de diverso sexo, consecuencias desiguales perjudiciales por el impacto diferenciado y desfavorable que tratamientos formalmente iguales o tratamientos razonablemente desiguales tienen 
Los principales rasgos que identifican este concepto es que se aplica a actos individuales de discriminación, directa o indirecta, e impone la paridad de trato y la interdicción absoluta de acto discriminatorio. ${ }^{9}$ En este sentido, la tutela frente a estos actos discriminatorios es de tipo negativa puesto que pretende castigar la discriminación una vez que se ha producido mediante una sanción y la reparación de sus efectos, fundamentalmente declarando la nulidad radical de los mismos. Por lo tanto, estaríamos más bien ante una obligación de no discriminar cuyo incumplimiento acarrea una sanción, sin que en ningún momento tenga la obligación de adoptar medidas de carácter promotor o preventivo. Tampoco sirve para las discriminaciones omisivas, esto es, aquellas consistentes en un no hacer. ${ }^{10}$

En este sentido, el artículo 17.1 del Estatuto de los Trabajadores (ET) Español es la mejor manifestación de ello: "Se entenderán nulos y sin efecto los preceptos reglamentarios, las cláusulas de los convenios colectivos, los pactos individuales y las decisiones unilaterales del empresario que den lugar en el empleo, así como en materia de retribuciones, jornada y demás condiciones de trabajo, a situaciones de discriminación directa o indirecta...”.

Más allá de estas manifestaciones del concepto clásico de discriminación, hay que hacer referencia a la noción de discriminación social, entendiendo por tal aquella producto de prácticas, prejuicios y usos sociales hacia colectivos que integran una sociedad. Como tal es manifestación del principio de igualdad sustancial o material, lo que supone que, aplicado a situaciones de desigualdad histórica de ciertos colectivos o grupos, se impone el restablecimiento del equilibrio social roto por medio de mecanismos más potentes que la mera eliminación de las manifestaciones concretas de la discriminación. De esta manera, este concepto se convierte en el fundamento de una tutela antidiscriminatoria positiva, que pretende el logro de la igualdad real a través de intervenciones especiales tendentes a lograr la igualdad de oportunidades rota por criterios de desigualdad social, realzando las diferencias realmente existentes entre los grupos tratados desigualmente, tal y como se señala en la ya citada Sentencia del TC 128/1987, de 7 de julio. ${ }^{11}$

sobre los trabajadores de uno y de otro sexo a causa de la diferencia de sexo". Respecto a la jurisprudencia del Tribunal de Justicia de la Unión Europea la sentencia de 13 de mayo de 1986, asunto 170/84, caso Bilka, es el referente en esta materia al ser la primera vez en que el Tribunal delimitan este concepto. Para comprender la importancia de estos conceptos véase Carmen Sáez Lara, Mujeres y mercado de trabajo. Las discriminaciones directas e indirectas (Madrid: CES, 1994), 33 y s.

9. Sierra Hernáiz, Acción positiva y empleo de la mujer, 16.

10. Por lo tanto, el problema radica en cuándo sea necesario exigir al empresario una conducta positiva lo que puede implicar la imposición de un deber de hacer el empresario. Véase al respecto, María Dolores Román de la Torre, Poder de dirección y contrato de trabajo (Valladolid: Ediciones Grapheus, 1992), 339.

11. Sierra Hernáiz, Acción positiva y empleo de la mujer, 21 y s. 
Ello implica la legitimidad de desigualdades introducidas para restablecer la igualdad socialmente ignorada, única fórmula de tutela activa que puede entenderse. Desde esta perspectiva las características de esta tutela son las siguientes. En primer lugar, no se exigen actos concretos discriminatorios sino que basta con el resultado final discriminatorio, puesto que se busca la abolición de los obstáculos existentes para la consecución de la igualdad sustancial desde una perspectiva promocional y no sancionadora. En segundo lugar, se atiende a la interacción política e histórica entre los individuos, los grupos y su entorno social, razón última de la situación de discriminación que se pretende combatir, por lo que no se exigen actos concretos e individualizables de conductas discriminatorias. Por último, la prueba de su existencia va unida a criterios de infrarrepresentación de los colectivos preteridos mediante la prueba estadística. ${ }^{12}$

\section{C) El PRINCIPIO DE IGUALDAD DE OPORTUNIDADES EN LA RELACIÓN LABORAL}

Unido a los conceptos de igualdad y no discriminación, el principio de igualdad de oportunidades en el ámbito laboral implica que cada trabajador o trabajadora disfrute de las mismas oportunidades a la hora de acceder al mercado de trabajo o permanecer en él por lo que habrá que eliminar todas las barreras que lo impidan. Dependiendo de a qué noción de igualdad se haga referencia, existe un principio de igualdad de oportunidades formal que implica que el factor de discriminación prohibido no puede ser un motivo legítimo de diferenciación, lo que supone la ausencia formal de barreras legales a la hora de acceder o permanecer en el mercado de trabajo por dicho motivo. ${ }^{13}$ Se trata, pues, de un perfil puramente negativo puesto que únicamente se plantea la consecución de la igualdad prohibiendo las discriminaciones formales sin recalar en las desigualdades debidas a los efectos persistentes de las discriminaciones ya superadas formalmente. Desde esta perspectiva será discriminatoria cualquier diferenciación por razón de sexo, salvo que exista una justificación razonable y objetiva que la legitime. ${ }^{14}$ La protección de la maternidad biológica de la trabajadora es un ejemplo de la excepción a este principio. ${ }^{15}$

12. Ibíd., 26 y s.

13. Ibíd., 20 y s.

14. Rodríguez-Piñero y Fernández López, Igualdad y discriminación, 60 y s.

15. Sobre esta cuestión véase Elisa Sierra Hernáiz, Prevención de riesgos laborales, embarazo de la trabajadora y lactancia natural (Pamplona: Aranzadi, 2016), 37 y s. 
En cambio, el principio de igualdad de oportunidades material va un paso más allá y permite la distribución desigualdad de ciertos bienes como forma de superar y eliminar los efectos de la discriminación social hacia determinados colectivos, poniendo especial énfasis en la consecución de la igualdad de oportunidades en el momento final y no en el inicio. Es más, y como expresión de una tutela antidiscriminatoria unilateral, con un sentido emancipador y corrector, admite que determinadas causas de discriminación sean el fundamento constitucional de un trato formalmente desigual puesto que es la causa última de las conductas discriminatorias que se pretende definitivamente erradicar y que impiden al colectivo femenino gozar de una verdadera igualdad de oportunidades en el empleo. ${ }^{16}$

\section{D) LAS MEDIDAS DE ACCión POSITIVA}

El concepto de acción positiva, como manifestación o instrumento de las políticas de igualdad de oportunidades material, hace referencia las medidas que pretenden aumentar la presencia de las mujeres en el mercado laboral primando la contratación, promoción o mantenimiento del empleo femenino. Se trataría, pues, de tratamientos preferenciales a favor de las trabajadoras entendiendo por tales las reservas de puesto de trabajo. Su finalidad es combatir los efectos de la discriminación social sobre la mano de obra femenina en el mercado laboral, actuando tanto en el lado de la oferta como en el de la demanda para garantizar su plena igualdad de oportunidades. ${ }^{17}$

Así delimitada sus principales características son las siguientes. En primer lugar, no es necesaria una especificación concreta del acto discriminatorio que se pretende combatir sino que la existencia de la discriminación se ha de probar por medio de la infrarrepresentación de las trabajadoras en el sector laboral donde se vaya a implantar la medida de acción positiva. En segundo lugar, el sexo de la trabajadora se convierte en el factor determinante a la hora de conceder el puesto de trabajo o la promoción. No puede olvidarse que las medidas de acción positiva se idean como el último instrumento para evitar que conductas discriminatorias arraigadas en la sociedad sigan perpetuándose ante el fracaso de las medidas reparadoras tradicionales. Y, en tercer lugar, tiene que estar perfectamente justificada su implantación, para lo cual han de superar el juicio de razonabilidad y proporcionalidad que impone el principio de igualdad ya que son una excepción al mismo. Para ello los parámetros que hay que utilizar son los de la igualdad sustancial y, a su vez, garantizar que en ningún caso se establecen privilegios o ventajas arbitrarias. Todo ello dota a dicho juicio de gran complejidad, que demanda un análisis exhaustivo de cada

16. Sierra Hernáiz, Acción positiva y empleo de la mujer, 10 y s.

17. Ibíd., 77-9. 
situación concreta en la que vaya a implantarse la acción positiva, no bastando la mera existencia de un contexto discriminatorio o una desigualdad numérica. ${ }^{18}$

\section{EL MARCO NORMATIVO Y LA INTERPRETACIÓN DE LOS TRIBUNALES DE LAS MEDIDAS DE ACCIÓN POSITIVA}

\section{A) El Derecho comunitario europeo y su aplicación EN EL ORDENAMIENTO JURÍDICO ESPAÑOL}

El concepto de acción positiva es deudor del derecho comunitario que define dichas medidas y fija sus límites a la hora de ser aplicadas en los países comunitarios. En este sentido, el artículo 141.4 Tratado de Funcionamiento de la Unión Europea que se remite el artículo 3 de la Directiva 54/2006, establece que:

Con objeto de garantizar en la práctica la plena igualdad entre hombres y mujeres en la vida laboral, el principio de igualdad de trato no impedirá a ningún Estado miembro mantener o adoptar medidas que ofrezcan ventajas concretas destinadas a facilitar al sexo menos representado el ejercicio de actividades profesionales o a evitar o compensar desventajas en sus carreras profesionales.

De esta redacción legal se desprende que las medidas de acción positiva son una excepción legítima al principio de igualdad de trato siempre y cuando sean medidas neutras, es decir, su establecimiento no se haga teniendo en cuenta el sexo de los destinatarios, sino solo si están o no infrarrepresentados. Por lo tanto, para el legislador comunitario su implantación como medidas en pos de la igualdad de oportunidades real depende exclusivamente de un factor objetivo de carácter numérico: la infrarrepresentación del sexo en la actividad o sector en el que se apliquen sin que, a priori, sus destinatarias puedan ser exclusivamente las trabajadoras.

El primer problema interpretativo surge de la delimitación de la acción positiva como una excepción a la igualdad de trato. En este caso, el legislador comunitario sí que está admitiendo que el sexo sea un factor legítimo de discriminación desde los parámetros de la igualdad real, siempre y cuando el sexo beneficiado esté infrarrepresentado, con independencia del origen de esta desigualdad numérica.

Por lo tanto, una vez fijadas su legitimación desde un punto de vista de legalidad constitucional, hay que enfrentarse al segundo problema que plantea la redacción le-

18. Ibíd., 84-5. 
gal: el requisito de la infrarrepresentación como elemento legitimador de la medida de acción positiva. Así, se exige que el sexo beneficiado esté menos representado -es importante resaltar que introduce el concepto de pertenencia a un grupo definido por el sexo para acceder a estas ventajas-, puesto que este factor -la infrarrepresentaciónes el que legitima en última instancia la aplicación de la medida de acción positiva. Ello implica que lo primero que habrá que hacer a la hora de su implantación es un estudio de su ámbito de aplicación para determinar si este requisito se cumple puesto que, en caso contrario, no tendrán cabida en el ordenamiento jurídico comunitario. Una vez constatado que se cumple dicho requisito, la medida de acción positiva será legítima, lo que podría plantear situaciones un tanto surrealistas, como tratamientos preferenciales para trabajadores varones en sectores en los que estén infrarrepresentados, como por ejemplo matronas, sin necesidad de que sea un colectivo socialmente e históricamente discriminado.

Respecto del ordenamiento jurídico español se sigue esta noción de acción positiva. En concreto, en el artículo 17.4 ET dispone que:

Sin perjuicio de lo dispuesto en los apartados anteriores, la negociación colectiva podrá establecer medidas de acción positiva para favorecer el acceso de las mujeres a todas las profesiones. A tal efecto podrá establecer reservas y preferencias en las condiciones de contratación de modo que, en igualdad de condiciones de idoneidad, tengan preferencia para ser contratadas las personas del sexo menos representado en el grupo o categoría profesional de que se trate.

Asimismo, la negociación colectiva podrá establecer este tipo de medidas en las condiciones de clasificación profesional, promoción y formación, de modo que, en igualdad de condiciones de idoneidad, tengan preferencia las personas del sexo menos representado para favorecer su acceso en el grupo, categoría profesional o puesto de trabajo de que se trate.

En cuanto a los ascensos, el artículo 24 ET establece que: "Los ascensos y la promoción profesional en la empresa se ajustarán a criterios y sistemas que tengan como objetivo garantizar la ausencia de discriminación directa o indirecta entre mujeres y hombres, pudiendo establecerse medidas de acción positiva dirigidas a eliminar o compensar situaciones de discriminación".

\section{B) La doctrina del Tribunal de Justicia DE LA UNIÓN EUROPEa SOBRE ACCIÓn POSITIVA}

Al igual que sucede con los conceptos de discriminación directa e indirecta, la delimitación legal de las acciones positivas en el ordenamiento jurídico europeo también es deudora de la doctrina del TJUE. En los fallos de este Tribunal se puede apreciar una 
evolución a la hora de analizar la acción positiva y establecer sus requisitos como una excepción al principio de igualdad de trato consagrado en el derecho comunitario. ${ }^{19}$

En este proceso dos han sido las sentencias fundamentales. Así, en el caso Kalanke el Tribunal entendió que la reserva de puestos de trabajo a favor de las mujeres no constituía una excepción legítima al principio de igualdad de trato ya que instauraba preferencias absolutas e incondicionales. ${ }^{20}$ Es a partir del caso Marschall cuando la perspectiva del Tribunal cambia al tener en cuenta la situación de discriminación social que afecta al colectivo femenino que de facto impide su igualdad de oportunidades real a través de su adecuado acceso al mercado laboral o progresión en la carrera profesional. ${ }^{21}$

Así, la doctrina jurisprudencial se puede resumir en los siguientes puntos.

El primero es que la acción positiva no constituya una preferencia absoluta e incondicional, para lo cual debe existir una cláusula de apertura que permita considerar las circunstancias particulares de cada caso y, excepcionalmente, denegar la preferencia a la mujer. Este requisito fue formulado en la Sentencia Marschall y su importancia radica en que ha servido para legitimar la adopción de medidas de acción positiva. Eso remarca, como se señaló en la Sentencia Badeck, la obligatoriedad de la acción positiva siempre que se respete la cláusula de apertura. ${ }^{22}$ En este sentido, la cláusula de apertura es el correctivo que el Derecho exige para la admisibilidad de estas medidas en el mercado de trabajo.

E1 TJUE no se pronuncia acerca del contenido de esta cláusula aunque de su doctrina se pueden deducir dos condiciones previas para su correcta aplicación: por un lado, que las candidaturas sean objeto de una apreciación objetiva que tengan en cuenta todos los criterios relativos a los candidatos de ambos sexos; y, por otro, que tales criterios no perjudiquen a las candidatas femeninas, esto es, no sean criterios discriminatorios. Así las cosas, cumplidas las condiciones de apreciación objetiva de

19. Sobre la evolución e impacto de la jurisprudencia comunitaria en la materia, véase: Elisa Sierra Hernáiz, "El concepto de medida de acción positiva en el ordenamiento jurídico comunitario y su aplicación al derecho español”, en Mario Santiago Juárez, coord., Acciones afirmativas (México: Consejo Nacional para prevenir la discriminación, 2011), 129 y s.

20. Caso Kalanke, de 17 de octubre de 1995, asunto c-450/93. Este fue el primer pronunciamiento judicial sobre la legalidad de las medidas de acción positiva en el derecho comunitario europeo y supuso su rechazo frontal a las mismas. La norma analizada fue la Directiva 76/207 que consagraba únicamente el principio de igualdad trato en su vertiente formal. Desde esta perspectiva el Tribunal no admitió el establecimiento de preferencias automáticas a favor de mujeres capacitadas en sectores en los que se encuentren infrarrepresentadas ya que con este tipo de medidas lo que realmente se perseguía era la igualdad de resultados a través de la paridad numérica.

21. Caso Marschall, de 11 de noviembre de 1997, asunto c-409/95.

22. Caso Badeck, de 28 de marzo de 2000, asunto c-158/97. 
méritos y no discriminación, si en el momento de la contratación o de la promoción se comprueba la existencia de alguna circunstancia determinante para otorgar el puesto al hombre, entonces este deberá ser contratado o promovido en lugar de la mujer. ${ }^{23}$

En segundo lugar, que la persona beneficiada pertenezca a un colectivo infrarrepresentado, siempre que esa menor presencia sea consecuencia de conductas discriminatorias para lo cual habrá de tener en consideración las características del ámbito de aplicación de la medida de acción positiva y las circunstancias específicas de cada sector o campo, tal y como se señala en el caso Badeck.

En tercer y último lugar, tal y como se señala en el caso Abrahamsson, se exige que los candidatos tengan méritos equivalentes o sensiblemente equivalentes para evitar vulnerar el principio de objetividad. Por consiguiente, no basta que la candidata tenga una capacitación suficiente para desempeñar las funciones del puesto de trabajo sino que sus méritos han de ser equivalentes o sensiblemente equivalentes a los de otros candidatos que concurran al mismo puesto de trabajo. Condición sine qua non es que existan criterios claros, precisos y objetivos para valorar los méritos de cada candidato al margen de valoraciones o criterios sexistas y discriminatorios. ${ }^{24}$

\section{LA COMPLEJA APLICACIÓN DE LA ACCIÓN POSITIVA EN LA RELACIÓN LABORAL A FAVOR DE LAS TRABAJADORAS}

La especial dificultad a la hora de adoptar estas medidas en el marco de la relación laboral es debida a diversas razones. ${ }^{25}$ En primer lugar, su configuración legal como excepción al principio de igualdad de trabajo y la referencia al sexo menos representando y la exigencia de una cláusula de apertura no ayudan en su implantación en la práctica. En segundo lugar, la propia complejidad de la relación laboral, ya que se

23. El Tribunal no se ha pronunciado sobre dichos requisitos, tan solo habla de motivos de rango superior en el Sentencia Badeck. No obstante es de suponer que su contenido estará unido al ejercicio de derechos fundamentales o principios del Estado Social que merezcan una protección igual a la de los principios que integran estas medidas. En relación con este aspecto, el caso Lommers, de 19 de marzo de 2002, asunto c-476/99, sigue esta vía al considerar la atención a los hijos pequeños como un factor legítimo que rompe la automaticidad de la medida a favor de las mujeres y que tiene en consideración factores personales que defiende un Estado Social de Derecho.

24. Caso Abrahamsson, de 6 de julio de 2000, asunto c-407/98.

25. En general sobre esta cuestión: Elisa Sierra Hernáiz, "La regulación del principio de igualdad de oportunidades en la Ley Orgánica de Igualdad como límite al poder de organización y dirección del empresario", en Elisa Sierra Hernáiz, dir., Eficiencia, igualdad y empresa. La aplicabilidad real a la empresa de la Ley Orgánica de Igualdad (Madrid: Civitas, 2011), 213 y s. 
trata de una relación de carácter privado que se asienta en un conflicto de intereses radicalmente opuestos, en este caso entre el derecho del trabajador a no ser discriminado y el ejercicio de los derechos consustanciales al poder de organización y dirección del empresario, exigiéndose una modelización recíproca entre ambos. ${ }^{26}$

En el caso que nos ocupa, el legislador español, al igual que el comunitario, ha establecido que el empresario no podrá discriminar a sus trabajadores, siendo un derecho básico de los trabajadores (art. 4.2.c (ET) cuya violación acarrea la nulidad radical del acto empresarial (art. 17.1. ET). Pues bien, si la nulidad es el límite más importante para el ejercicio de los poderes empresariales para garantizar la igualdad de oportunidades de los trabajadores cabría preguntarse si es el más eficaz visto que las persistentes diferencias entre los géneros en el mercado laboral.

Además, hay que tener en cuenta que el actual sistema de relaciones laborales se fundamenta sobre los principios de la economía libre de mercado y el derecho a la libertad de empresa consagrados en el art. $38 \mathrm{CE}$ y la autonomía de la voluntad de las partes manifestada en el contrato de trabajo. ${ }^{27}$ Este reconocimiento supone que el empresario tiene libertad para organizar su empresa conforme a las finalidades que él mismo establezca, siendo el poder de organización y dirección del empresario uno de los instrumentos para ejecutar dicha libertad empresarial. ${ }^{28} \mathrm{Si}$ bien tiene límites al mismo, la prohibición de discriminación es una de ellas tal y como ha sido expuesto, en la actual configuración no se le impone ninguna obligación de hacer, entre ellas el trato igual entre sus trabajadores.

En concreto, respecto de la acción positiva, el modelo es absolutamente voluntario, sin que existan incentivos reales de cara a su implantación, lo que hace que sean hoy por hoy medidas residuales, por no decir inexistentes, en el ordenamiento laboral español a favor de las trabajadoras. La situación cambia cuando el colectivo beneficiado por los tratamientos preferenciales en el mercado laboral son jóvenes, parados

26. María Dolores Román de la Torre, Poder de dirección y contrato de trabajo, 301 y s.

27. En general, sobre esta cuestión véase: María Dolores Santos Fernández, El contrato de trabajo como limite al poder del empresario (Albacete: Bomarzo, 2005).

28. A su vez, cuando se discute sobre la posibilidad de establecer límites al poder de dirección del empresario se identifica como un todo dicho poder cuando se necesario precisar a qué manifestaciones concretas nos estamos refiriendo, ya que no es lo mismo hablar de límites al poder disciplinario que al poder de organización, aunque ambos sean expresión del mismo principio, a saber, el poder de dirección del empresario, consustancial al contrato de trabajo como manifestación de la dependencia, que es uno de los requisitos esenciales para la existencia de la relación laboral. En relación con esta cuestión todavía se puede hacer una puntualización en cuanto a la diferencia que existe entre el poder disciplinario del empresario, cuya prueba es mucho más accesible, del poder de organización y dirección del empresario, cuando produzca actos discriminatorios de prueba mucho más difícil. Roman de la Torre, Poder de dirección y contrato de trabajo, 301 y s. 
de larga duración o trabajadores con discapacidad ya que la puesta en marcha de este tipo medidas no se cuestionan, al menos con la misma intensidad que se aplica si son trabajadoras, ni encuentran tantos obstáculos, ni legales ni sociales.

Un ejemplo de ello en el ordenamiento jurídico español es el Real Decreto Legislativo 1/2013, de 29 de noviembre 8, BOE No. 289), por el que se aprueba el Texto Refundido de la Ley General derechos de las personas con discapacidad y de su inclusión social que a lo largo de su articulado reconoce diversas medidas de acción positiva para este colectivo sin que se exijan los requisitos de aplicación para las trabajadoras. Es más, existe una cuota de reserva que impone que las empresas con 50 o más trabajadores han de reservar el 2 por 100 de los puestos de trabajo para las personas con discapacidad sin que hasta el momento se haya cuestionado su constitucionalidad. ${ }^{29}$

En tercer lugar, en torno al concepto de acción positiva existe cierta confusión terminológica ya que se tiende a identificar como tal un conjunto de medidas como derechos de protección de la maternidad o de la conciliación de la vida laboral o familiar que en sí mismos nada tienen que ver con la acción positiva y que se dirigen más bien a garantizar la igualdad de trato. Estos derechos forman parte de un modelo tradicional de igualdad y no discriminación que hasta el momento, a pesar de los avances logrados, se ha mostrado insuficiente para lograr la igualdad de oportunidades real.

\section{A MODO DE CONCLUSIÓN}

La aplicación de medidas de acción positiva a favor de las trabajadoras en la relación laboral ha generado una gran desconfianza, lo que ha llevado a imponer una serie de requisitos legales y jurisprudenciales que dificultan in extremis su aplicación en la práctica. A pesar de que es entendible de alguna manera el recelo que puedan provocar, en gran medida motivado por el desconocimiento de su verdadero significado, ello no puede suponer su olvido como mecanismos de la igualdad de oportunidades real.

En este sentido, la necesidad de establecer este tipo de medidas sigue estando plenamente justificada ante la persistente situación de desigualdad de la mujer en el mercado laboral en diferentes situaciones: la diferencia salarial; la menor tasa de empleo y la mayor tasa de desempleo; la segregación horizontal y vertical del empleo feme-

29. Respecto al ordenamiento jurídico ecuatoriano el artículo 11.2 de la Constitución ecuatoriana establece que: "El Estado adoptará medidas de acción afirmativa que promuevan la igualdad real en favor de los titulares de derechos que se encuentren en situación de desigualdad”. Sobre la aplicación de las medidas de acción positiva en Ecuador véase: Macarena Bahamonde, "La falsa concepción de la acción positiva a favor de las mujeres como medida de discriminación directa”, USFQ Law Review. 17 y s. Disponible en 〈https://www.usfq.edu.ec/publicaciones/lawreview/Documents/edicion003/law_review_004.pdf〉. 
nino, etc., son motivos suficientes para aplicarlas, siempre y cuando estén plenamente justificadas y respondan a criterios objetivos de necesidad unidos a acabar con las situaciones apenas descritas. ${ }^{30}$ Por ello, es necesario volver a la vertiente promotora y preventiva del principio de igualdad material para entender el rol fundamental que estas medidas pueden desempeñar para acabar con los efectos de la discriminación social que aún padecen las trabajadoras. En este sentido el artículo 45 de la LOI sí que establece una obligación de hacer para los empresarios:

Las empresas están obligadas a respetar la igualdad de trato y de oportunidades en el ámbito laboral y, con esta finalidad, deberán adoptar medidas dirigidas a evitar cualquier tipo de discriminación laboral entre mujeres y hombres, medidas que deberán negociar, y en su caso acordar, con los representantes legales de los trabajadores en la forma que se determine en la legislación laboral.

Con todo, el principal problema de las medidas de acción positiva se encuentra delimitación legal del ordenamiento jurídico comunitario, puesto que la acción positiva no es un instrumento para corregir situaciones de infrarrepresentación de los sexos en el ámbito laboral, ya que su objetivo no es conseguir una igualdad numérica sino acabar con la discriminación social que se manifiesta precisamente a través de la prueba estadística dada la dificultad de identificar los efectos concretos de este concepto de discriminación. Es aquí dónde radica el principal error de la normativa comunitaria y del Tribunal de Justicia de la Unión Europea que está impidiendo, en la práctica de los hechos, que estas medidas se apliquen en el ámbito de la relación laboral.

\section{BIBLIOGRAFÍA}

Bahamonde, Macarena. "La falsa concepción de la acción positiva a favor de las mujeres como medida de discriminación directa”. USFQ Law Review. Disponible en 〈https://www.usfq. edu.ec/publicaciones/lawreview/Documents/edicion003/law_review_004.pdf.

Fabregat Monfort, Gemma. Las medidas de acción positiva: la posibilidad de una nueva tutela antidiscriminatoria. Valencia: Tirant lo Blanch, 2009.

Giles Carnero, Rosa María. "La jurisprudencia del Tribunal de Justicia en materia de igualdad entre hombres y mujeres. Especial referencia al concepto de acción positiva”. En M. MoraRuiz, dir., Formación y objeto del derecho antidiscriminatorio de género: perspectiva sistemática de la igualdad desde el Derecho público. Barcelona: Atelier, 2010.

30. Véase los datos del Informe La participación laboral de las mujeres en España (Madrid: Consejo Económico y Social, 2017). 
Informe La participación laboral de las mujeres en España. Madrid: Consejo Económico y Social, 2017.

Lousada Arochena, José Fernando. El derecho fundamental a la igualdad efectiva de mujeres y hombres: fundamentos del derecho a la igualdad de género y, en especial, su aplicación en el derecho del trabajo y de la seguridad social. Valencia: Tirant lo Blanch, 2014.

Mateu Carruana, María Josefina. "Igualdad de trato y no discriminación entre mujeres y hombres en el empleo: significado y alcance de la Directiva 2006/54/CE”. En A. Sempere Navarro y M. Areta Martínez, Cuestiones actuales sobre derecho social comunitario. Murcia: Laborum, 2009.

Mendoza Navas, Natividad. “Acciones positivas: instrumento para eliminar las diferencias entre hombres y mujeres en el ámbito de las relaciones laborales”. En Martín López, dir., La igualdad de género desde la perspectiva social, jurídica y económica. Madrid: Civitas, 2014.

Nogueira Guastivino, Magdalena. "El principio de igualdad y no discriminación en las relaciones laborales: perspectiva constitucional reciente". Lan Harremanak: Revista de Relaciones Laborales, No. 25 (2011).

Rodríguez-Piñero, Miguel, y María Fernanda Fernández López. Igualdad y discriminación. Madrid: Tecnos, 1986.

Román de la Torre, María Dolores. Poder de dirección y contrato de trabajo. Valladolid: Ediciones Grapheus, 1992.

Sáez Lara, Carmen. Mujeres y mercado de trabajo. Las discriminaciones directas e indirectas. Madrid: Consejo Económico y Social, 1994.

Santos Fernández, María Dolores. El contrato de trabajo como límite al poder del empresario. Albacete: Bomarzo, 2005.

Sierra Hernáiz, Elisa. Acción positiva y empleo de la mujer. Madrid: Consejo Económico y Social, 1999.

---. "El concepto de medida de acción positiva en el ordenamiento jurídico comunitario y su aplicación al derecho español”. En Mario Santiago Juárez, coord., Acciones afirmativas. México: Consejo Nacional para prevenir la discriminación, 2011.

---. "La regulación del principio de igualdad de oportunidades en la Ley Orgánica de Igualdad como límite al poder de organización y dirección del empresario”. En Elisa Sierra Hernáiz, dir., Eficiencia, igualdad y empresa. La aplicabilidad real a la empresa de la Ley Orgánica de Igualdad. Madrid: Civitas: 2011.

---. Prevención de riesgos laborales, embarazo de la trabajadora y lactancia natural. Pamplona: Aranzadi, 2016. 